


\section{Article Artículo Paper La participación desde 2 el Trabajo Social: una aproximación conceptual en el marco de los servicios sociales de atención básica}

\section{Marta Arranz \\ Montull ${ }^{1}$}

Joan Casas ${ }^{2}$

\section{Resumen}

El concepto de participación se ha considerado definitorio de la disciplina del Trabajo Social. No obstante, la literatura científica señala su carácter altamente polifacético y ambiguo. El presente artículo pretende aproximarse conceptualmente a la participación dentro del marco de los servicios sociales de atención básica en el contexto de la ciudad de Barcelona. A través del uso de técnicas de corte cualitativo (entrevistas individuales y grupos de discusión), se han analizado las narrativas de diferentes agentes sociales (familias atendidas por los servicios sociales, profesionales y cargos de gestión) para poder abordar detallada y contextualmente la complejidad del concepto. Los resultados obtenidos nos confirman su heterogeneidad y, a su vez, nos ayudan a pensar la participación en los servicios sociales a través de tres variables: los niveles de participación, las características principales y

1. Doctora por la Universitat de Barcelona. Unitat de Formació i Recerca (UFR)- Escola de Treball Social. Universitat de Barcelona

2. Graduado en Trabajo Social. Unitat de Formació i Recerca (UFR)- Escola de Treball Social. Universitat de Barcelona 
las condiciones. A grandes rasgos, se destaca la amplia aprobación ontológica que recibe la participación por parte de los distintos agentes entrevistados en relación con aquellas prácticas de intervención social y de planificación de los servicios. Sin embargo, también se ponen de manifiesto las dificultades para desarrollar dichos procesos participativos por la falta de un consenso conceptual operativo. Finalmente, se pone de relieve que la participación de las personas usuarias requiere una relación más transparente entre la administración y la ciudadanía, así como el reconocimiento de las desigualdades sociales y las relaciones de poder subyacentes.

Palabras clave: Participación, Trabajo Social, Servicios Sociales

\begin{abstract}
The concept of participation has been considered to define the discipline of Social Work. However, the scientific literature points out its highly multifaceted and ambiguous characteristics. This article aims to conceptually approach participation within the framework of social services in the context of the city of Barcelona. Through the use of qualitative techniques (individual interviews and focus groups), the narratives of different social actors (families served by social services, professionals and management) have been analyzed in order to contextually address in detail the complexity of the concept. The results obtained confirm its heterogeneity and, in turn, help us think about participation in social services through three variables: levels of participation, main characteristics and conditions. Broadly speaking, the wide ontological approval that participation receives from the different social actors interviewed in relation to those practices of social intervention and planning of services stands out. However, it also highlights the difficulties in developing such participatory processes due to the lack of an agreed operational concept and effective political support until now. Finally, it shows that the participation of users requires a more transparent relationship between the administration and the public, as well as the acknowledgement of social inequalities and underlying power relations.
\end{abstract}

Keywords: Participation, Social Work, Social Services 


\section{Introducción}

La participación se ha mostrado tradicionalmente como uno de los conceptos clave en la disciplina del Trabajo Social. A pesar de ello, muestra cierta ambigüedad debido a los diferentes contextos en los que se aplica, en ocasiones con objetivos y terminologías específicas. En el presente artículo se pretende conceptualizar el término dentro del marco de los servicios sociales de atención básica, los cuales, como se indica en la Ley 12/2007, de 11 de octubre, de servicios sociales, "son el primer nivel del sistema público de servicios sociales y la garantía de más proximidad a los usuarios y a los ámbitos familiar y social" (art. 16.1). Además, tienen como finalidad prevenir, dar respuesta, atender y capacitar a las personas a lo largo de sus ciclos vitales (Matulic y Fustier, 2011: 320).

Los servicios sociales están organizados territorialmente y se estructuran en equipos multidisciplinares que proporcionan atención individual, familiar y colectiva. Su perspectiva de promoción de las capacidades se centra en fomentar que las personas atendidas sean protagonistas de sus propias situaciones de vida, potenciando acciones preventivas y de carácter relacional, y ofreciendo una atención prioritaria a las situaciones de vulnerabilidad social y dependencia. Además, los entes locales tienen un papel central en el diseño de políticas de proximidad; su principal ventaja recae en el hecho de que permiten ajustar el sistema a las necesidades de su entorno, detectar estas necesidades, pero, también, identificar y dinamizar los recursos de la propia comunidad (Subirats, 2007, citado en Matulic y Fustier, 2011: 320).

Por ello, es preciso interrogarse sobre el alcance de los cambios en los procesos de intervención y sobre la participación y el protagonismo de las personas en estos procesos. De esta forma, se plantea investigar sobre la participación de las familias en los procesos de intervención profesional dentro de los servicios sociales de atención básica. Así pues, la presente publicación muestra los resultados preliminares obtenidos de la primera fase de la investigación, "[Re]Pensemos la participación de las familias: Diagnosis y propuestas de intervención en los servicios de atención básica de las personas en la ciudad de Barcelona" (20192022), la cual tiene como objetivo general:

Construir un diagnóstico y propuestas de cambio, colectivamente desde las miradas de los diferentes actores, sobre la participación de las familias en los itinerarios de atención en los servicios sociales de atención básica. 
De este modo, en esta publicación se destacan los resultados más relevantes referentes en la aproximación conceptual de la participación en el marco de los servicios sociales. Los resultados se obtuvieron entre octubre y diciembre de 2019 y fueron analizados de diciembre de 2019 a enero de 2020.

\section{La participación en la intervención social}

El concepto participación es complejo y constituye, por un lado, un punto de inflexión en el debate político y de la sociedad y, por otro lado, un impacto directo en las políticas sociales municipales. La participación es un elemento consustancial en nuestro contexto relacional, con lo que requiere la interacción reflexiva de los diferentes agentes sociales, ya que aporta infinidad de beneficios en la dinámica organizacional y comunitaria (Pastor, 2013; 2008; 2006).

En el contexto de las políticas sociales, la participación es "una herramienta fundamental para mejorar el diseño, evaluar los servicios y detectar las necesidades reales de la población" (Subirats, 2007: 57). No obstante, esta herramienta requiere de mecanismos que favorezcan los procesos participativos y la creación de procesos de decisión y gestión descentralizada y compartida. De esta forma, se mejora el funcionamiento de las instituciones creando un clima de entendimiento y confianza ciudadana (Pastor, 2013) y se genera capital social potenciando los sentimientos comunitarios (Carrasquilla y Seidel, 2005).

La sociedad actual y las realidades emergentes impulsan la necesidad de diseñar estrategias de desarrollo del territorio local (Martínez Román, 2000: 336). Por ello, la inclusión social y el desarrollo local son dimensiones vinculadas al Trabajo Social Comunitario, el cual promueve estrategias de análisis e intervención integrada y sostenible en el ámbito municipal (Pastor, 2006). A su vez, el proceso comunitario es un proceso participativo, ya que "sin participación no hay proceso, no hay cambios reales o, mejor dicho, los cambios siempre serán el producto de las decisiones de otros” (Marchioni, 1999: 16).

El método de trabajo de investigación participativa fortalece la identificación de necesidades, los procesos de autogestión y el impulso para el ejercicio de acciones cooperativas que permitan la transformación de las situaciones (Lo, 2021; Alonso, 2002). En esta misma línea, existen sólidos argumentos para involucrar los conocimientos de los usuarios 
y usuarias de los servicios en el proceso de construcción de la teoría del Trabajo Social (Beresford, 2000).

Siguiendo a Gaitán (2003), la participación se puede poner en práctica de diferentes formas e intensidades promoviendo el acceso de las personas a la toma de decisiones. Por lo tanto, la integración de la participación en la toma de decisiones será determinante en el modelo y la perspectiva de la intervención profesional, la cual debe estar estructurada en tres pasos fundamentales: 1) querer, tomar conciencia del problema; 2) saber, reconocerse con capacidades y comprometerse para llevar a cabo acciones que transformen la realidad y; 3) poder, favorecer contextos de promoción de la creatividad y la innovación.

No obstante, algunos datos ponen de relieve una importante disyuntiva entre la retórica de la participación de las personas de distintos servicios sociales y la tendencia y capacidad para adoptar tales prácticas en el terreno. La falta de una transformación adecuada de los sistemas lleva a que la retórica de la participación de las personas de distintos servicios haya recaído históricamente en los individuos (Bee, et al., 2015). Actualmente se reconoce ampliamente la necesidad de que las políticas y prácticas de participación de los usuarios tengan en cuenta las desigualdades sociales para facilitar su participación e influencia significativas, ya que estas son las que contribuyen a su silenciamiento y exclusión (Lewis, 2014).

\section{Metodología}

La construcción de un concepto de participación, situado en el marco de los servicios sociales de atención básica de la ciudad de Barcelona, implica profundizar en las formas de participación de las familias en el diseño de los servicios y en las diferentes fases de los procesos de atención e intervención.

Para ello, la metodología utilizada, de corte cualitativo, tiene un enfoque de investigación participativa, en el que son reconocidos todos los agentes implicados en el contexto del estudio (Beresford, 2013). Diferentes estudios evidencian que la participación de la ciudadanía en los proyectos de investigación, desde una perspectiva de investigación participativa, es una excelente vía para acceder a grupos de población de difícil acceso (Newell y South, 2009). Las perspectivas de investigación participativas sobre las prácticas 
de intervención social generan acciones basadas en los códigos éticos profesionales y en los principios que enmarcan las leyes de los servicios sociales.

Las personas participantes han sido profesionales que ocupan un cargo de gestión en diferentes niveles de la estructura de los servicios sociales del Ayuntamiento de Barcelona, profesionales involucrados en la atención básica y personas o familias vinculadas a los servicios sociales.

Para la recogida de datos se han utilizado la entrevista y los grupos de discusión como herramientas cualitativas de recogida de información. La nomenclatura de cada instrumento es la siguiente: entrevistas individuales (EI), grupos de discusión (GD) y un grupo motor (GM).

En la investigación han participado un total de 126 personas. Por un lado, se realizaron un total de 39 entrevistas individuales, de las cuales 9 fueron a cargos de gestión, 15 a profesiones y 13 familias participantes. Por otro lado, se realizaron 10 grupos con un total de 87 personas, en los que estaban los tres grupos de participantes.

El análisis de los resultados se ha realizado a través de las transcripciones de las entrevistas y de los grupos de discusión. La información obtenida se ha codificado utilizando el programa Atlas-ti, versión 8 para su posterior análisis.

\section{Imagen 1: Número de participantes y técnicas}

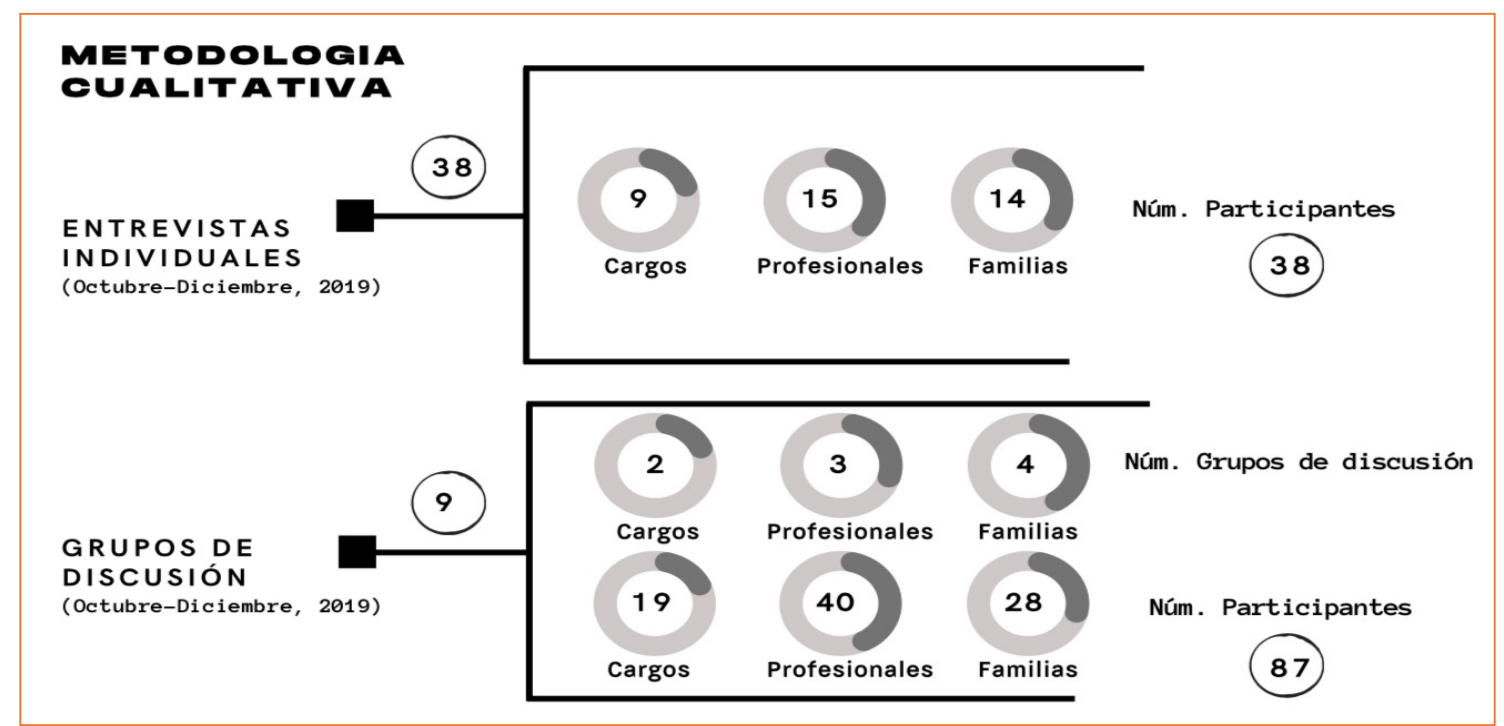

Fuente: XXX citar primer artículo introducción 


\section{Resultados}

\subsection{Conceptualización de la participación en los servicios sociales}

El Comité de Protección Social de la Comisión Europea (2010) dictaminó que la participación es uno de los principios de calidad de los servicios sociales, sugiriendo a los proveedores de servicios promover la participación de las personas usuarias. En este sentido, los servicios sociales municipales deben facilitar el acceso y la participación de las personas en su globalidad y no sólo como sujetos pasivos o beneficiarios de actividades y prestaciones.

Es necesario pasar de una participación pasiva a una participación sustantiva y real de las familias durante los procesos de intervención, dando soporte al trabajo de los y las profesionales (Pastor, 2009). No obstante, Lewis (2014) señala que en la participación de los usuarios y usuarias puede suceder que se pasen por alto sus opiniones dentro de los ámbitos de la política y la planificación. Esta autora, citando a Bourdieu (1992), ilustra cómo las prácticas políticas discursivas de los usuarios y usuarias de servicios suelen estar reguladas y acotadas por estructuras de poder preexistentes que delimitan su influencia en el campo. Los y las profesionales participantes coinciden en señalar la dificultad existente para lograr dicha horizontalidad en una organización de tipo piramidal.

Esta participación es muy limitada, muy controlada... Porque es que la propia institución es jerárquica. Entonces cuando nosotros pedimos, por ejemplo, participar en las decisiones de lo que nos afecta, también tenemos dificultades porque hay una jerarquía [...]. Y cuesta encontrar espacios donde estemos políticos, jefes territoriales, directores, trabajadores y familias. Es difícil encontrar eso (GD- Cargos de gestión)

Subirats (2007: 124) añade que, para diseñar un sistema de servicios sociales que fomente la participación y la corresponsabilidad entre los agentes, se hace necesario, por un lado, la distribución de las responsabilidades entre los distintos agentes que desarrollan acciones, programas o servicios sociales. Esto implica, en muchos casos, mejorar la coordinación, hasta ahora débil, entre el sector público, el privado y el voluntariado. Por otro lado, se refiere a la importancia que se debería conceder al conjunto de agentes no lucrativos que tradicionalmente han desarrollado actuaciones de intervención social, así como a la ciuda- 
danía en general respecto de la participación en el diseño y la elaboración de programas, actuaciones y servicios en el marco de los servicios sociales.

La participación cuenta con un amplio reconocimiento y apoyo ideológico por parte de los agentes sociales vinculados al sistema de servicios sociales. No obstante, ha estado comúnmente obstaculizada por una potente combinación de retrasos conceptuales y operativos, así como por una falta de entendimiento compartido con respecto al concepto (Bee et al., 2015). El trabajo de campo realizado en una muestra de personas vinculadas personal o profesionalmente a los servicios sociales de atención básica de la ciudad de Barcelona, ha puesto de manifiesto la amplitud de significados en torno al concepto de participación en estos ámbitos. Para algunas personas que ocupan cargos de gestión participar implica la capacidad de colaborar, incluyendo a las personas usuarias para que sean "agentes de cambio".

Por participación, lo primero que se me ocurre, es la capacidad, ¿̇no?, de poder colaborar todos y con las personas para que sean agentes activos de cambio, que ellos participen en el diseño de los servicios. Los recursos que hay, que puedan responder a las necesidades reales de la gente. (EI Cargo de gestión)

Además, la mayoría de las familias indican que participar es formar "parte de"; es una "oportunidad" a la que han sido invitadas, sentirse integradas dentro de un proceso, de un servicio o de un proyecto. Implica también poder expresar una opinión y que ésta sea escuchada y considerada. Las entrevistas realizadas ponen de manifiesto la importancia de la participación para los propios usuarios y usuarias, tanto en los procesos de definición de necesidades como en los procesos de definición de las formas de satisfacerlas.

La participación es la oportunidad que tienes para participar en lo que te invitan a participar. Es dar tu opinión, básicamente. Y que ésta sea tenida en cuenta de la manera que sea. La participación es la oportunidad de decir y opinar en relación a que se requiere. (EI-Familia)

La Figura 1 muestra un esquema que resume los elementos que articulan la participación de acuerdo con los y las participantes de estas dos etapas de investigación. La identificación de una serie de niveles, características significativas y algunas condiciones necesarias nos ayuda a delimitar una aproximación a las conceptualizaciones situadas de participación. 


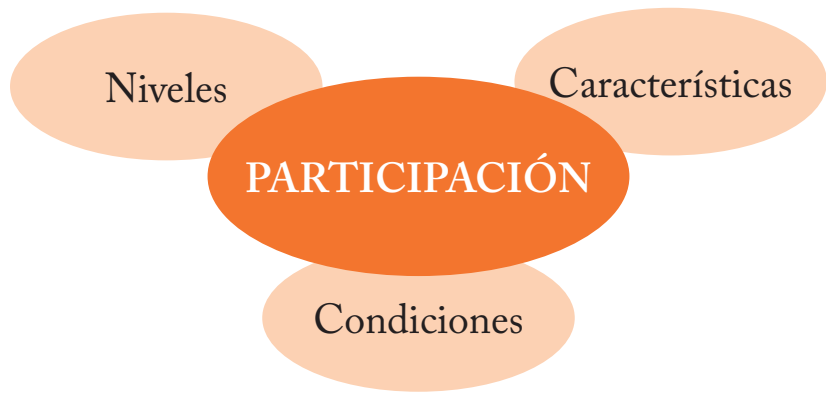

Fuente: Elaboración propia

\subsubsection{Niveles}

Se hace necesario diferenciar, en primer lugar, de qué tipo de participación hablamos cuando nos referimos a participar en los servicios sociales ¿Hablamos de la participación de las familias en términos de ser agentes activos de sus procesos de cambio y de mejora psicosocial? ¿Hablamos de la participación de las familias en el diseño de los servicios y de las prestaciones de las cuales ellas u otras familias serán beneficiarias?. En segundo lugar, también conviene hablar del carácter gradual de estas participaciones, entendiendo que no en todos los casos tendrán la misma intensidad e influencia.

En el marco de los servicios sociales, la Ley 12/2007 establece lo que se podría considerar un primer nivel de participación situada a la que algunos autores y autoras se han referido como participación cívica (Pelegrí, 2011: 230) o participación ciudadana (Alberich y Espadas, 2014). Esta se sitúa en los principios de una administración relacional y con los sectores afectados o implicados en los servicios sociales y parte de la premisa de que las decisiones relativas al sistema de servicios sociales deben tomarse con la participación de los ciudadanos siempre que sea posible (art.46). La finalidad de esta participación es integrarla en los procesos de toma de decisión para adecuar el sistema de servicios sociales a las necesidades de las personas y a su diversidad (Matulic y Fustier, 2011: 320).

Los objetivos de la participación son la implicación de toda la sociedad en los asuntos sociales, la prevención de la fragmentación social, la innovación en la prestación de los servicios y el reforzamiento de las redes sociales de apoyo (Art. 47.2, Ley 12/2007, de 11 de octubre, de servicios sociales). 
Para este fin, existen dos canales prioritarios para su articulación: unos órganos de participación y unos procesos participativos que se tienen que aplicar en la planificación, gestión y evaluación de los servicios sociales.

\section{Tabla 1. Canales prioritarios para articular la participación en los procesos de toma} de decisiones relativas al sistema de servicios sociales

\section{\begin{tabular}{l|l} 
Órganos de participación & Procesos participativos
\end{tabular}}

- Consejo general de servicios sociales

Incluyen tres fases:

- Consejos territoriales de servicios sociales

1. Fase de información del proyecto

- Consejos municipales o supramunicipales de servicios sociales para el que se pide la participación

2. Fase de debate ciudadano

- Consejos de centro

3. Fase de retorno de resultados

Fuente: Elaboración propia en base a Pelegrí (2011: 230-231)

En el marco de la intervención social, parecería que la participación resulta más fácil y factible en las actividades grupales y comunitarias que en la atención individualizada, en la cual parece que su articulación sea más difícil de lograr. Ejemplo de ello son las formas de participación social en el ámbito de las personas mayores identificadas por Douglas et al., (2017): 1) Las conexiones sociales; 2) La participación social informal y; 3 ) El voluntariado. No obstante, algunos autores y autoras señalan que la prudencia o tendencia conservadora de las administraciones ha llevado a propiciar el trabajo individualizado. Las administraciones no han querido apostar por acciones sociales que vehicularan la participación (Fernández et al., 2016) y estas han ido quedando paulatinamente marginadas en las políticas sociales (Alberich y Espadas, 2014).

Pero ¿qué tipo de participación se puede dar en el marco de un proceso de intervención social individual y familiar? Los itinerarios de atención y tratamiento social con familias, sin duda los más frecuentes en el contexto de los servicios sociales objeto de análisis, comprenden espacios para la participación activa de las personas usuarias en dichos procesos. En este segundo nivel de participación, un nivel más micro o centrado en procesos individuales y familiares, se incorpora la noción de empowerment. El trabajo que parte de esta noción, la cual proviene del trabajo comunitario, se basa en la capacitación 
de las personas atendidas (así como de las familias y grupos) para que se configuren como agentes activos en su proceso de mejora. Boehm y Staples (citados en Fernández et al., 2016) destacan como función empoderadora el hecho de que tanto unos agentes como otros, tengan una participación activa en las políticas sociales, pero también en la toma de decisiones respecto sus procesos de cambio.

Desde la administración si queremos personas empoderadas también debemos escucharlas, pero también tenemos que aceptar sus críticas y sus quejas ¿Realmente estamos reconociendo esta capacidad de las personas de coger este poder? (GDCargos de gestión)

Los y las profesionales manifiestan que en la fase del diseño del plan de trabajo individualizado es imprescindible contar con la participación de las personas ya que, sin el acuerdo sobre dicho plan, este no se podrá llevar a cabo. No obstante, se considera que la participación también debe promoverse durante todo el proceso de intervención. El análisis de las narrativas recoge e ilustra esta cuestión:

La participación para mi entra en juego desde la primera entrevista, en la parte del estudio de cómo le explicas quién eres, qué es lo que vas a hacer, que es lo que va a pasar, que es lo que ella cree de esa situación, que es lo que crees tú que te pueda rebatir lo que tu piensas. (GD- Profesionales)

En ambos niveles de participación categorizados, la participación se presenta con un carácter plenamente gradual y no absoluto: "participar es como un proceso, no como una finalidad en sí misma sino como un proceso para llegar, en el que hay diferentes grados" (EI- Cargo de gestión). En este sentido, algunos autores y autoras han categorizado las formas de participación de mayor a menor implicación y frecuencia. Primero, y con cierta frecuencia, la información, después (en menos casos) la consulta y debate, lo que implica un diálogo entre administración y ciudadanos, y sólo en contadas experiencias, la cogestión o toma de decisiones conjunta (Alberich y Espadas, 2014). Así pues, las formas más categóricas de participación, aunque también las menos frecuentes, implican el trabajo en común en un plano de igualdad (Gaitán, 2003). 


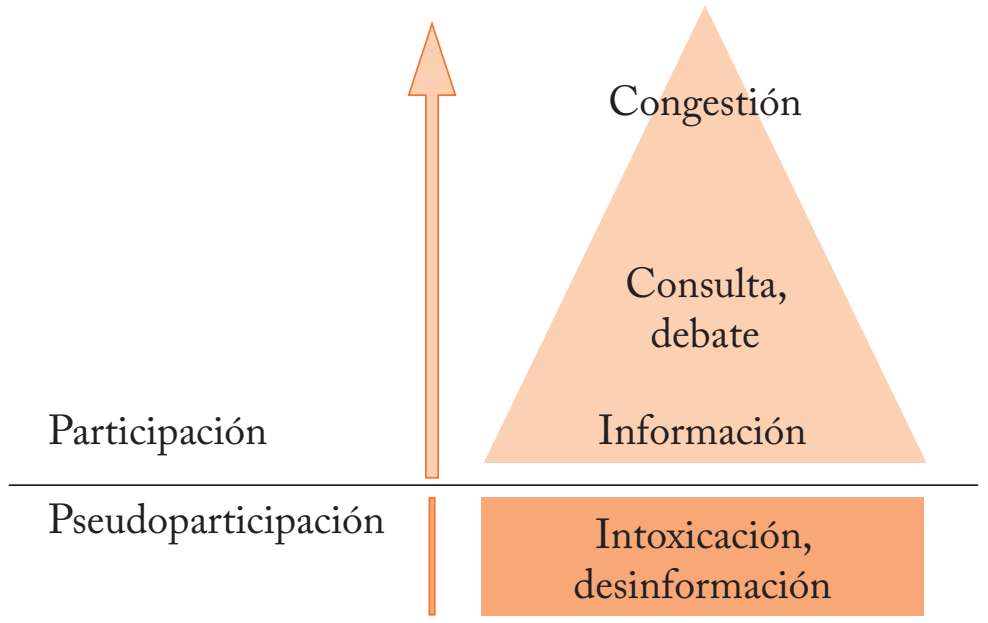

Fuente: Elaboración propia en base a Alberich y Espadas (2014: 25).

Otro ejemplo de este carácter gradual de la participación viene dado por la clasificación taxonómica que realizan Piškur et al., (2013) adaptándola de Levasseur et al., (2004).

\section{Tabla 2. Taxonomía para actividades sociales basadas en los niveles de implicación}

\begin{tabular}{|l|l|}
\hline Niveles & Descripción de los niveles de implicación/intervención \\
\hline Primer Nivel & Hacer una actividad de preparación para conectar con otras personas \\
\hline Segundo Nivel & Estar rodeado de otros \\
\hline Tercer Nivel & Interactuar con otros sin contacto físico \\
\hline Cuarto Nivel & Hacer una actividad con otros \\
\hline Quinto Nivel & Ayudar a otros \\
\hline Sexto Nivel & Contribuir a la comunidad \\
\hline
\end{tabular}

Fuente: Piškur et al. (2013) adaptada de Levasseur et al. (2004)

\subsubsection{Características principales}

Pastor (2008), tomando de referencia el enfoque de las necesidades humanas de Doyal y Gough (1994) y del desarrollo a escala humana de Manfred Max-Neff (1994), considera la participación como una necesidad humana que requiere un valor estratégico al intervenir 
directa y transversalmente, optimizando el acceso a la satisfacción de las demás necesidades. Diversos estudios, por ejemplo en el ámbito de las personas mayores, han encontrado asociaciones positivas entre la participación social y la salud, a través de sus mecanismos de apoyo social y cohesión con la comunidad en general (Douglas, et al., 2017).

La participación es un elemento fundamental en el conjunto de valores del Trabajo Social y se considera definitorio de la misma disciplina (Pastor, 2008). Los avances en el campo del Trabajo Social y la atención social para socializar su discurso y práctica apuntan a un enfoque alternativo para la futura política y práctica de bienestar basada en la participación plena y equitativa de sus destinatarios (Beresford, 2000).

Los y las profesionales que participaron en las primeras fases del estudio consideran la participación como un medio y no como una respuesta ni como una finalidad en sí misma.

Participar es formar parte [...] Se está hablando de participación desde el mismo momento que formas parte de, quiero decir que no es el resultado final que tu consigas sino simplemente el momento de estar allí. Estás participando de un contexto. (GD- Profesionales)

La participación de las familias vinculadas a los servicios sociales está estrechamente relacionada con la intervención profesional, y en concreto, con la relación que se establece entre los y las profesionales y las familias (Lo, 2021). De acuerdo con la opinión de los y las participantes, para lograr que las personas participen en sus propios procesos, es preciso el establecimiento de un vínculo. Esto es lo que permitirá que se construyan relaciones de seguridad y confianza y, sólo cuando dichas relaciones se generan, puede existir la participación.

La participación de las familias vinculadas a los servicios sociales es importante para las y los profesionales, pero existen factores como la edad, las barreras idiomáticas o culturales que pueden obstaculizarla, por lo que es preciso diseñar distintas estrategias para fomentarla en todos sus niveles.

Un niño de 10 años no puede participar de la misma manera que un señor de 80, porque no tiene las herramientas, no tiene la facilidad de comunicación, no tiene una cier- 
ta experiencia... No lo hará igual, pero puede participar. Esta es la diferencia, debemos buscar qué estrategias son las idóneas para que participe un niño de 10 años y cuáles son las idóneas para que participe un señor de 80. (EI Cargo de gestión)

Sin embargo, las personas participantes en los tres grupos de discusión de los diferentes agentes (familias, profesionales y cargos de gestión) coinciden en que el nivel de motivación y no la etapa de vida en la que se encuentre, es lo que hace que se impliquen o no en un proyecto grupal o en su plan de trabajo individual.

Yo no creo que la participación dependa de la edad, porque si tienes interés por el tema participarán más o menos. Y sobre todo si te invitan y te sientes interpelada todavía más. (EI- Familia)

Touhami (2020), en un artículo sobre participación y conocimientos de las personas a quienes se dirigen las políticas, hace el ejercicio de identificar una serie de obstáculos que dificultan la participación en el contexto institucional a diferentes niveles:

Tabla 3. Obstáculos que dificultan la participación en el contexto institucional a diferentes niveles.

\begin{tabular}{|l|l|l|}
\multicolumn{1}{|c|}{ Personas afectadas } & \multicolumn{1}{|c|}{ Equipos profesionales } & \multicolumn{1}{c|}{ Instituciones } \\
Autocensura, desconfianza, & Gestión de la urgencia, peso & Efectos de la “clausura" con el \\
hostilidad hacia los & de la rutina profesional e & mundo exterior que limitan la \\
trabajadores y las & inercia de la vida cotidiana; & introducción de innovaciones, \\
trabajadoras sociales, & desconocimiento del tema & herencia de la cultura de "la \\
efectos de la vergüenza, & por falta de formación e & ayudantía”y de la relación de \\
falta de autoestima, etc. & información; estereotipos & "ventanilla", no-priorización \\
& y prejuicios en torno al & del asunto por parte de \\
acceso a la participación; & las direcciones, existencia \\
resistencia, reconocida o no, & de consejos participativos \\
a la idea de conceder poder & "fantasmas" sin ninguna \\
a las personas afectadas, etc. & autoridad, etc. \\
\hline
\end{tabular}

Fuente: Elaboración propia en base a Touhami (2020: 50). 
En esta misma línea, De la Red y Barranco (2014) señalan que el aumento de la demanda urgente a los servicios sociales, la gestión de las dificultades y la prioridad de cubrir las necesidades básicas derivan a la tramitación masiva de prestaciones asistenciales y reducen de forma significativa la intervención profesional basada en la promoción de capacidades y a la participación de las poblaciones.

Por otra parte, también se pone en duda la existencia de una verdadera voluntad política para fomentar la participación. Para algunos de los y las profesionales la participación es una estrategia política para legitimar decisiones preexistentes.

Es como cuando nos han hecho participar en procesos de cambio y luego resulta que esto ya estaba previsto previamente. Pero claro, vas al proceso participativo porque va muy bien y está muy "guay”. Pero, ¿podemos decir que hemos participado? [...] Para mí es una falsa participación. (EI Profesional)

Se afirma que a veces se produce una "falsa" participación, o pseudoparticipación (Alberich y Espadas, 2014), de las personas o las familias cuando está mediatizada o no pueden incidir en los procesos de toma de decisiones reales sobre sus problemas o necesidades sociales. Se debería, por tanto, fomentar la participación real de las familias y personas usuarias, y que esta pueda llegar a influir en la implementación y mejora de las políticas sociales.

Te quieren hacer ver que participas, pero en realidad no participamos en la toma de decisiones, nunca. Esto en la Ley de Servicios Sociales o en la Ley de no sé dónde tiene un nombre... cuando tú participas, pero tu voto no cuenta se convierte en algo testimonial. (EI- Familia)

\subsubsection{Condiciones}

$\mathrm{El}$ análisis de los niveles de participación en los servicios sociales, sus características y sus obstáculos nos lleva a afirmar la necesidad de que esta se base en una relación transparente e igualitaria entre la administración y la ciudadanía. Resulta difícil imaginar el desarrollo de una política de participación de las personas vinculadas a los servicios sociales fuera de un contexto político participativo. La transparencia de esa relación implica que las aportaciones de la ciudadanía puedan llegar a modificar las acciones de la administración. Está claro que los falsos proyectos participativos se desenmascaran rápidamente y sólo pueden generar desencanto y desafección entre los servidores públicos y la ciudadanía (Al- 
berich y Espadas, 2014). Por lo tanto, la honestidad, la transparencia y la voluntad política real serán las condiciones fundamentales para el desarrollo de proyectos participativos en cualquiera de los ámbitos que conciernen a los servicios públicos (Pastor, 2009).

No sólo la administración es responsable del éxito de la implementación de las políticas participativas, también es necesario que las familias se impliquen en sus procesos de cambio; se impliquen de forma activa y asuman el valor de poder influir realmente en las cuestiones que les conciernen. El proceso que vincula a los agentes necesita pedagogía, transparencia y grandes dosis de generosidad, no en vano, se trata de una retrocesión de poder (Marchioni, 1999).

Para mí participar es que la persona o la familia realmente esté implicada en su proceso de cambio, cuando no lo está, sólo hace uso de los servicios sociales. Para mí la diferencia es ésta: hacer uso o realmente estar participando y ser consciente de que no todo el mundo es consciente de su problemática. (EI Profesional)

Algunas profesionales expresan la necesidad de tener una determinada actitud o predisposición participativa, señalando que está relacionada con el conocimiento sobre cuáles van a ser los beneficios que reporte la participación, sobre aquello que se va a obtener y también con lo que se puede aportar (Howe, 1999). Según Bee et al., (2015), la posibilidad de que existan barreras de actitud no se descarta, pero está claro que se puede hacer mucho más a nivel organizativo y político para permitir la participación de los usuarios y usuarias.

Además, se puso de relieve la manifestación de las familias en que no siempre se quiere participar en proyectos grupales o comunitarios o en el plan de trabajo individual, y que se debe respetar esta como una posible opción de las personas o familias: "También existe el derecho a no querer participar y esto no quiere decir que no se me deba ofrecer, sino que sencillamente no quiero participar." (EI- Familia)

Existe consenso sobre considerar la importancia del rol del profesional. Por un lado, el o la profesional debe escuchar a las personas vinculadas a los servicios sociales, necesita explorar y conocer el significado que para ellas tiene la participación en sus propios procesos, ya que los significados pueden ser diferentes para ambas partes. Además, se debe tener en cuenta que, en algunas ocasiones, las personas no puedan o no deseen participar. 
A veces hay personas que están en unos momentos vitales que no pueden tomar decisiones o no pueden hacer mucho. Y los tenemos que acompañar también en este proceso, y también esto es participación. (GD- Profesionales).

Por otro lado, para el ejercicio de la participación, sobre todo para los y las profesionales, estaría relacionada con las estrategias para su fomento, la participación no debe estar sujeta a sus imposiciones, tal como señalan las siguientes narrativas:

Reflexionar en qué posibilidades le damos a que pueda ser la parte activa de esa participación sin imposiciones porque al final siempre son imposiciones, condiciones y siguiendo las dinámicas que se establecen cuando entras al centro y ya hay la dinámica establecida que cuesta. (GD- Profesionales)

En la atención individualizada siguen predominando las relaciones verticales y consecuentemente unas relaciones de poder desiguales. De ahí la importancia de trabajar de manera más igualitaria entre el o la profesional y las familias vinculadas a los servicios sociales, pudiendo facilitar de esta forma la implementación de estrategias para consolidar la participación. Pero el establecimiento de un vínculo relacional sólido y confiable, entre las familias y la profesional, no es una tarea fácil. Desde el primer momento requiere empatía con las familias y clarificar la misión y la función del y de la trabajadora social (Howe, 1999).

Las familias que han participado en las dos primeras fases del estudio afirman que generalmente se establecen vínculos muy positivos con los y las profesionales y eso les permite no sentirse solas, perseguidas o juzgadas, como es señalado en las entrevistas realizadas.

Entras con miedo, pero ellos te hacen sentir tan bien, te hacen sentir que te conocen de años, te hacen sentir que están en tu piel, te sientes bien, tranquila. Te puedes expresar, te entienden lo que tú les dices, a veces con la mirada ya saben qué es lo que queremos, qué es lo que sentimos. (EI-Familia)

Por otro lado, los y las profesionales consideran que algunas familias perciben los servicios sociales como un distribuidor de recursos y ayudas y desde esa posición es difícil promover su participación. 
Hay familias que se implican y vienen. Están las familias que tienen, para mí, servicios sociales como un trabajo de "me levanto y voy a trabajar". "Pues vengo a servicios sociales a buscar las ayudas" [...] Y luego está la familia de "házmelo todo", "tramítame esto". O ahora lo que se ha puesto de moda es "me tienes que dar un piso, tú obligación es darme un piso, por ley me lo tienes que dar (EI Profesional)

Finalmente, otro factor que subrayan los y las profesionales, está relacionado con la dificultad que puede suponer la participación en las grandes ciudades a causa del acceso y el estigma aún latente en la población.

"Yo creo que otro tema que es vital, lo que comentábamos antes, abrir los centros al barrio. Que no sea “allí es dónde van los pobres”, “allí es donde va la gente que tiene problemas" no. (...). (GD- Profesionales)

\section{Reflexiones finales}

En este artículo se ha realizado un primer análisis sobre el concepto de la participación en los servicios sociales que deberá ser completado a lo largo de la investigación marco, ampliando su profundidad y poniendo en relación nuevas fuentes teóricas de referencia. Sin embargo, en estos resultados emergen nuevas aproximaciones conceptuales que nos pueden ayudar a pensarla y reivindicarla.

La participación de la ciudadanía en las decisiones que le concierne es la base de la democracia participativa. Aunque cuenta con una gran aprobación por motivos filosóficos, ideológicos y ontológicos, la planificación que involucra a las personas usuarias de determinados servicios está mal definida y carece de un apoyo efectivo para la implementación (Bee et al., 2015). Para que ésta sea real y efectiva, la información y la capacidad de decisión han de propiciar una relación horizontal entre la administración y los y las ciudadanas, así como reconocer las desigualdades sociales y las relaciones de poder preexistentes entre los y las diferentes agentes implicadas (Lewis, 2014).

Se ha identificado que la participación en los servicios sociales de atención básica se constituye como un proceso y no como una finalidad en sí misma. Además, se estructura en 
dos grandes niveles. Un primer nivel se enmarca en una participación cívica o ciudadana, la cual corresponde con los procesos de decisión para adecuar los servicios sociales a las necesidades de las personas. En cambio, el segundo nivel hace referencia a la implicación de las familias en sus procesos de atención social a través de su capacitación y empoderamiento.

En otra de las reflexiones que emergen del análisis cualitativo se señala que, en ocasiones, los simulacros participativos (o la pseudoparticipación) desmotivan a los y las profesionales de los servicios públicos y a las personas que están vinculadas a ellos. La participación de las personas en los servicios sociales, especialmente en el diseño y adecuación de los mismos, aún es un proyecto por desarrollar que requiere de una relación más transparente entre administración y ciudadanía. Además, es necesaria la tarea pedagógica que modifique prácticas obsoletas de los servicios sociales y que sea capaz de modificar ciertas aproximaciones de los y las ciudadanas a los servicios sociales.

Como es visible, en algunas situaciones resulta difícil mantener una relación igualitaria entre los y las profesionales y las personas que requieren sus servicios. Los datos apuntan a que las relaciones en el interior de los servicios públicos son de tipo piramidal y están sujetas a cambios de orientación política a corto plazo.

Para hacer un uso adecuado del proceso participativo es necesario el cumplimiento de una serie de condiciones, las cuales, además de una voluntad política e institucional, se basan en los valores actitudinales y en una predisposición participativa del trabajador/a social y/o de cualquier profesional que trabaja en el mismo equipo interdisciplinar de servicios sociales. Entre ellos deben estar presentes la escucha activa, la libertad de expresión sin imposiciones, la horizontalidad respecto a los y las profesionales y facilitar orientación e información del propio proceso participativo.

La participación acontece una necesidad humana, la cual se convierte en un elemento fundamental para la práctica del Trabajo Social y de las disciplinas de intervención social afines, ya que se convierte en un medio para la intervención social.

Finalmente, el posicionamiento de los y las profesionales y de los cargos de gestión, es un elemento indispensable, ya que influye de forma directa en la promoción de la participación en los servicios sociales. Por ello, es necesario que exista una convicción unánime 
sobre la participación de las familias entre los y las profesionales, para que se puedan promover cambios significativos.

\section{Bibliografía}

Alberich, T. y Espadas, A. (2014). Democracia, participación ciudadana y funciones del trabajo social. Trabajo Social Global. Revista de Investigaciones en Intervención Social, 4 (6), 3-30.

Alonso; R. (2002): Intervención comunitaria en el Trabajo Social. Proyecto Docente a Cátedra. Valencia. Roneo.

Bee, P., Brooks, H., Fraser, C., \& Lovell, K. (2015). Professional perspectives on service user and carer involvement in mental health care planning: a qualitative study. International journal of nursing studies, 52(12), 1834-1845.

Beresford, P. (2000). Service Users' Knowledges and Social Work Theory: Conflict or Collaboration? The British Journal of Social Work, 30(4), 489-503.

Beresford, P. (2013). From 'other' to involved: user involvement in research: an emerging paradigm. Nordic Social Work Research, (3)2, pp. 139-148.

Carrasquilla, Ma . C., y Seidel, S. E. (2005): La participación ciudadana: vía para la integración de las personas inmigrantes. Rutas y caminos, Agrupación de Desarrollo NEXOS.

De la Red Vega, N., y Barranco Expósito, C. (2014). Trabajo Social y participación en las políticas sociales. AZARBE, Revista Internacional De Trabajo Social Y Bienestar, (3). https://revistas.um.es/azarbe/article/view/198341

Douglas, H; Georgiou, A \& Westbrook, J. (2017). Social participation as an indicator of successful aging: an overview of concepts and their associations with health. Australian Health Review, 41, 455-462. DOI: http://dx.doi.org/10.1071/AH16038 
Fernández, J. (coord) (2016). La intervenció amb famílies des del Treball Social. Grup de Recerca i Innovació en el Treball Social (GRITS). Departament de Treball Social i Serveis Socials. Universitat de Barcelona. Col.legi Oficial de Treball Social de Catalunya: Barcelona.

Gaitán, L. (2003): “Ciudadanía, participación y Trabajo Social”, ponencia presentada en Inauguración Curso 2003/2004 E.U.T.S. de Murcia.

Howe, D. (1999). Dando sentido a la práctica: una introducción a la teoría del trabajo social. Granada: Maristán.

Ley 12/2007, de 11 de octubre, de Servicios Sociales, BOE núm. 266, de 06 de noviembre de 2007.

Lo, C.K.M.; Yu, L.; Cho, Y.W.; Chan, K.L. (2021). A Qualitative Study of Practitioners' Views on Family Involvement in Treatment Process of Adolescent Internet Addiction. Int. J. Environ. Res. Public Health, 18, 86.

Lewis, L. (2014). User involvement in mental health services: a case of power over discourse. Sociological Research Online, 19(1), 1-15.

Marchioni, M. (1999). Comunidad, participación y desarrollo. Madrid: Editorial Popular, 27.

Martinez Román, Ma .A. (2000): “Pobreza, exclusión social y cultura de paz”. En J. A. Díaz y Ma. J. Salvador (Coords.): Nuevas perspectivas de los servicios sociales. UNED. Madrid. 315 - 344 .

Matulic, V., Fustier, N. (2011). Els serveis socials bàsics. En: X. Pelegrí (Ed.), Els serveis socials a Catalunya: Aportacions per al seu estudi (pp.319-338). Lleida: Edicions de la Universitat de Lleida.

Newell, C. J. \& South, J. (2009). Participating in community research: exploring the experiences of lay researchers in Bradford Community. WorkEFFamily, 12(1), pp. 75-89. 
Pastor, E. (2006). La participación ciudadana en los servicios sociales municipales de la Región de Murcia. (Tesis Doctoral). Universidad de Murcia, Murcia. https://digitum.um.es/digitum/handle/10201/148

Pastor, E. (2008). E1 Trabajo Social ante la encrucijada de la Participación Ciudadana en el ámbito local. De lo simbólico a lo sustantivo. En: Trabajo Social Hoy no 55. Madrid: Colegio Oficial de Diplomados en Trabajo Social y AA. SS. de Madrid.

Pastor, E. (2009). Participación y gestión de las políticas sociales municipales.Murcia: Editum.

Pastor, E. (2013). Ciudadanía y participación en contextos de fractura y exclusión social. Pedagogía Social. Revista Interuniversitaria, 2013, 22, pp. 91-103. http://www.upo.es/revistas/index.php/pedagogia_social/

Pelegrí, X. (2011). Organització dels serveis socials. En: X. Pelegrí (Ed.), Els serveis socials a Catalunya: Aportacions per al seu estudi (pp.319-338). Lleida: Edicions de la Universitat de Lleida.

Piškur, B.; Daniëls, R.; Jongmans, M.J; Ketelaar, M.; Smeets, R.; Norton, M. \& Beurskens, A. (2013). Participation and social participation: are they distinct concepts? Clinical Rehabilitation, Vol. 28(3) 211-220. DOI: 10.1177/0269215513499029.

Subirats, J. (2007). Los Servicios Sociales de Atención Primaria ante el cambio social. Madrid:Ministerio de Trabajo y Asuntos Sociales.

Touhami, S. (2020). Participació i coneixements dels "usuaris" i les persones a qui s'adrecen les polítiques. Pedagogia i Treball Social. Revista de Ciències Socials Aplicades. Vol. 9. Núm. 2: 47-54 
